\title{
Dietary practices and beliefs of patients with older-onset inflammatory bowel disease: A prospective study from the United Kingdom
}

\author{
B. Crooks ${ }^{1,3}$, J.K. Limdi ${ }^{2,4}$ and J.T. McLaughlin ${ }^{1,3,4}$ \\ ${ }^{1}$ The University of Manchester, Division of Diabetes, Endocrinology and Gastroenterology, Manchester, UK, \\ ${ }^{2}$ The Pennine Acute Hospitals NHS Trust, Section of IBD - Division of Gastroenterology, Jericho Road, Bury, \\ Manchester, UK, \\ ${ }^{3}$ Salford Royal NHS Foundation Trust, Department of Gastroenterology, Salford, UK and \\ ${ }^{4}$ Manchester Academic Health Science Centre, Manchester, UK
}

In an aging population, $25-35 \%$ of those with inflammatory bowel disease (IBD) are over 60 years old. A third of these are diagnosed with IBD at or over the age of 60 , so-called "older-onset" IBD ${ }^{(1,2)}$. Despite epidemiological associations implicating diet in IBD aetiology ${ }^{(3)}$, virtually no studies exist exploring the dietary practices and beliefs of patients with older-onset IBD. Elderly patients with IBD are at increased risk of nutritional deficiency and associated complications making it all the more important to understand the dietary habits and self-imposed restrictions of this group.

A prospective, cross-sectional, questionnaire-based study is being conducted across NHS Trusts within the UK. Two-hundred patients with older-onset IBD will be recruited to complete a questionnaire regarding demographics and dietary practices. Here we provide an interim analysis of the data collected from the first 55 patients.

Mean patient age was 72 years, 53\% were male, 95\% Caucasian, 65\% had ulcerative colitis and 35\% had Crohn's disease. Mean disease duration was 6 years. $35 \%$ of patients felt diet was an initiating factor in their IBD and this was based on their own experience $(79 \%)$ or advice from a gastroenterologist $(42 \%)$, dietitian $(21 \%)$ or GP $(21 \%)$ in the majority of cases. Just under a third of patients believed that diet could trigger a relapse of their disease with the most common triggers identified as raw fruit and vegetables $(56 \%)$, spicy foods $(44 \%)$, fatty foods $(38 \%)$ and milk products $(31 \%)$.

$64 \%$ of patients avoided certain foods to prevent relapse. The most commonly avoided foods were spicy (83\%) and fatty foods $(71 \%)$, carbonated drinks $(63 \%)$, red meat $(49 \%)$, raw fruit and vegetables $(49 \%)$ and alcohol $(49 \%)$. Half of patients reported being able to find specific advice regarding dietary recommendations in IBD, and $70 \%$ of these used the internet to obtain this advice. Almost a third of patients avoided eating the same menu as their family at least some of the time to prevent relapse of their IBD and $35 \%$ of patients avoided eating out. Nearly one in five patients had tried a specific exclusionary diet to help manage their disease, most commonly gluten or lactose free.

Despite a low proportion of patients implicating diet in the initiation of IBD or its relapse, a significant number continue to restrict their diet. This, along with co-existing frailty, comorbidities and polypharmacy, may put this group of patients at increased risk of nutritional deficiency and its associated complications. Improved knowledge of the dietary habits of those with "older-onset" IBD will allow healthcare professionals to identify those who may be putting themselves at risk and offer appropriate dietary interventions. A larger dataset will be presented at conference proceedings.

Support provided by Takeda

1. Sturm A et al. (2017) J Crohns Colitis 11, 263-273.

2. Jeuring SF et al. (2016) Inflamm Bowel Dis 22, 1425-1434.

3 . Ng SC et al. (2018) Lancet 390, 2769-2778. 